\title{
Overview of Zika virus (ZIKV) infection in regards to the Brazilian epidemic
}

\author{
S.N. Slavov ${ }^{1,2}$, K.K. Otaguiri ${ }^{1,3}$, S. Kashima ${ }^{1,3}$ and D.T. Covas ${ }^{1,2}$ \\ ${ }^{1}$ Hemocentro de Ribeirão Preto, Faculdade de Medicina de Ribeirão Preto, Universidade de São Paulo, Ribeirão Preto, SP, Brasil \\ ${ }^{2}$ Departamento de Clínica Médica, Faculdade de Medicina de Ribeirão Preto, Universidade de São Paulo, \\ Ribeirão Preto, SP, Brasil \\ ${ }^{3}$ Departamento de Análises Clínicas, Toxicológicas e Bromatológicas, Faculdade de Ciências Farmacêuticas, \\ Universidade de São Paulo, Ribeirão Preto, SP, Brasil
}

\begin{abstract}
Zika virus (ZIKV), a mosquito-borne flavivirus, belongs to the Flaviviridae family, genus Flavivirus. ZIKV was initially isolated in 1947 from a sentinel monkey in the Zika forest, Uganda. Little clinical importance was attributed to ZIKV, once only few symptomatic cases were reported in some African and Southeast Asiatic countries. This situation changed in 2007, when a large outbreak was registered on the Yap Island, Micronesia, caused by the Asian ZIKV lineage. Between 2013 and 2014 , ZIKV spread explosively and caused many outbreaks in different islands of the Southern Pacific Ocean and in 2015 autochthonous transmission was reported in Brazil. Currently, Brazil is the country with the highest number of ZIKV-positive cases in Latin America. Moreover, for the first time after the discovery of ZIKV, the Brazilian scientists are studying the possibility for the virus to cause severe congenital infection related to microcephaly and serious birth defects due to the time-spatial coincidence of the alarming increase of newborns with microcephaly and the Brazilian ZIKV epidemic. The present review summarizes recent information for ZIKV epidemiology, clinical picture, transmission, diagnosis and the consequences of this emerging virus in Brazil.
\end{abstract}

Key words: Zika virus; ZIKV; Transmission; Epidemiology; Congenital infection; Brazil

\section{Introduction}

Zika virus (ZIKV) was isolated in 1947 from a febrile sentinel Rhesus monkey from the Zika forest on the banks of lake Victoria, Uganda (1). The virus was not linked to a human infection until 1954, when ZIKV was detected in three patients during an outbreak of jaundice in East Nigeria (2). Almost at the same time, ZIKV has been isolated from Aedes (Stegomya) africanus mosquitoes, in Africa, and Aedes aegypti, in Southeast Asia $(3,4)$. From 1954 to 1993 serologic evidence for ZIKV infection had been reported from various African countries including Kenya (5), Sierra Leone (6), Gabon (7,8), Ivory Coast (9), Central African Republic (10), and Senegal (11). By this time, in Asia, ZIKV has been detected in individuals with acute fever from Central Java and Lombok Island, Indonesia $(12,13)$. This early serological evidence demonstrated that ZIKV circulates in some countries of SubSaharan Africa and Southeast Asia with relatively low number of symptomatic cases ( $<10$ official cases). The situation changed in 2007, when an outbreak of a Dengue virus (DENV)-like disease characterized by rash, conjunctivitis and arthralgia was registered on the Yap Island,
Micronesia (Figure 1). Rapid DENV tests suggested that it was the etiological agent for that outbreak and the collected samples were sent to the Centers for Disease Control and Prevention (CDC) Arbovirus Diagnostic Laboratory for viral confirmation. The performed tests with Flaviviridae genus consensus primers demonstrated sequences sharing 90\% nucleotide identity with ZIKV. Therefore, the Yap Island epidemic was caused by ZIKV and not DENV and, specifically, by the ZIKV Asian lineage (14). The Yap Island outbreak demonstrated that ZIKV was not restricted to Africa and Southeast Asia and could cause epidemics outside its habitual occurrence. Nevertheless, Micronesia is geographically close to the countries of Southeast Asia, where ZIKV is endemic and it could be supposed that travelers or trade have introduced the virus in Oceania. Surprisingly, between 2013 and 2014, ZIKV continued its spread through the Southern Pacific and caused its largest outbreak in French Polynesia with an estimated occurrence of 19,000 cases in various islands from this region. The identified genotype, similarly to the epidemic in the Yap Island, 
belonged to the Asiatic lineage and its global expansion was suspected (15). Moreover, the Asian lineage expanded rapidly to many South Pacific islands and outbreaks were reported from New Caledonia, the Cook Islands and the Easter Island, Chile (16) (Figure 1).

The first official report for ZIKV autochthonous transmission in Brazil was documented in March 2015 in the city of Natal, in the Northeast part of the country. The patients presented with a benign disease characterized by mild fever, macopapular rash, headache, conjunctivitis, arthralgia and edema, similar to the symptoms caused by DENV, however all were DENV-negative (17). After confirmation of autochthonous transmission of ZIKV in Brazil, it was speculated that ZIKV entered the country during the 2014 World Football Championship; however, no countries endemic for ZIKV infection were competing in this event. The most probable ZIKV introduction in Brazil seems to have occurred during the Va'a World Sprint Championship, where competitors from countries with ZIKV outbreaks, i.e., French Polynesia, New Caledonia, Cook Islands, and Easter Island, have been participating (18). This event coincided with the first report of autochthonous transmission of ZIKV in Brazil and with the confirmation of the Asiatic lineage of the circulating strain. Currently, Brazil is the country with the highest number of ZIKV-positive cases in Latin America with circulation of the virus in almost the entire national territory.

\section{Viral characteristics}

ZIKV belongs to the Flaviviridae family, genus Flavivirus, closely related to the Spondweni virus (19). Therefore, ZIKV has the typical flavivirus organization of the virion with an icosahedral, enveloped particle. The genome is a singlestranded, positive-sense RNA of approximately $11 \mathrm{~kb}$. The genomic organization follows that of the flaviviruses with two flanking non-coding regions (NCR), i.e. 59 and 39 , and a long open reading frame encoding a single polyprotein: (NCR59)-C-prM-E-NS1-NS2A-NS2BNS3-NS4A-NS4BNS5-(NCR39). The polyprotein is cleaved into capsid (C), precursor of membrane (prM), envelope (E) and seven nonstructural proteins (NS) (20). The E protein, a major viral envelope protein, is involved in receptor binding and membrane fusion. The domain III of the $E$ protein contains different antigenic epitopes that may be important targets for serological assays, neutralizing antibodies, and vaccines (21). Worldwide dissemination of ZIKV is thought to be due to loss of the N154 glycosylation site of the E protein, thus permitting viral adaptation to a broader range of mosquito vectors (20).

\section{ZIKV genotypes}

Phylogenetic studies suggest that ZIKV circulating strains can be separated into two clades: African and Asiatic. The African strains are comprised of two groups: the MR766 prototype cluster (isolated in Uganda) and the Nigerian cluster. The Asian clade, on the other hand, is also comprised of two groups: the Micronesian and the Malaysian strains. ZIKV strains found in Western Africa (Ivory Coast and Senegal) were distributed between both African clusters suggesting that both lineages of the African clade are circulating in Western Africa. The NS5 and $E$ genomic regions have the highest phylogenetic signal content and are more suitable for the reconstitution of ZIKV phylogenetic history (20). The overall genetic divergence between the African and Asiatic clades is relatively low, $<11.7 \%$. Therefore, conserved regions among the worldwide circulating ZIKV strains can be used for the design of molecular detection assays and ZIKV differentiation from other flaviviral infections. Genotyping data support the hypothesis that the genotype causing the Yap epidemic originated in Southeast Asia. It is unknown how the virus spread to Oceania. One of the hypotheses suggests that wind-blown mosquitoes can travel several hundred kilometers through the ocean and may have introduced ZIKV in islands closely situated to Southeast Asia. However, due to the great distances in the Pacific Ocean it is more likely that the virus was introduced in Oceania as a result of tourists or trade activities originating in regions endemic for the ZIKV Asian lineage (22).

\section{ZIKV transmission}

ZIKV is an arbovirus primarily transmitted by mosquito vectors. The first isolates of ZIKV have been obtained from Aedes (Stegomyia) africanus (3), in Africa, and from Aedes aegypti (4), in the Malayan peninsula, Southeast Asia, implicating that the mosquitoes of the Aedes genus are the principal transmitting vectors. Consequently, ZIKV has been isolated from different Aedes mosquitoes in Africa including Ae. furcifer, Ae. luteocephalus, Ae. taylori, Ae. dalzieli, Ae. opok, Ae. vittatus, Ae. jamoti, Ae. flavicollis, Ae. grahami, Ae. taeniarostris, Ae. tarsalis, Ae fowleri, Ae. metallicus, Ae. minutus, Ae. neoafricanus, Ae. albopictus (22-24). In Africa, ZIKV has been also isolated from mosquitoes belonging to the genera Anopheles ( $A n$. coustani, An. gambiae), Mansonia (Ma. uniformis) and Culex (Cx. perfuscus) $(22,23)$. This wide range of arthropod vectors in Africa that can host ZIKV demonstrates that the virus is well adapted to different mosquito genera, which can play an important role during sylvatic and urban ZIKV cycles in Africa (Figure 2).

Despite that many mosquito species have been identified as ZIKV hosts, its reservoirs in nature remain unknown. Seroprevalence of ZIKV has been demonstrated in Old World non-human primates of the Cercopithecus genus (Guenons), including Cercopithecus aethiops (African green monkey), Cercopithecus ascanis schmidti (Red-tailed monkey), Cercopithecus mona denti (Mona monkey), and Cercopithecus albigena johnstoni and also the Colobus genus (Colobus abyssincius, Mantled guereza) (22). ZIKV has also been isolated from Cerocopithecus aethiops and Erythrocebus patas monkey species (22) (Figure 2). 


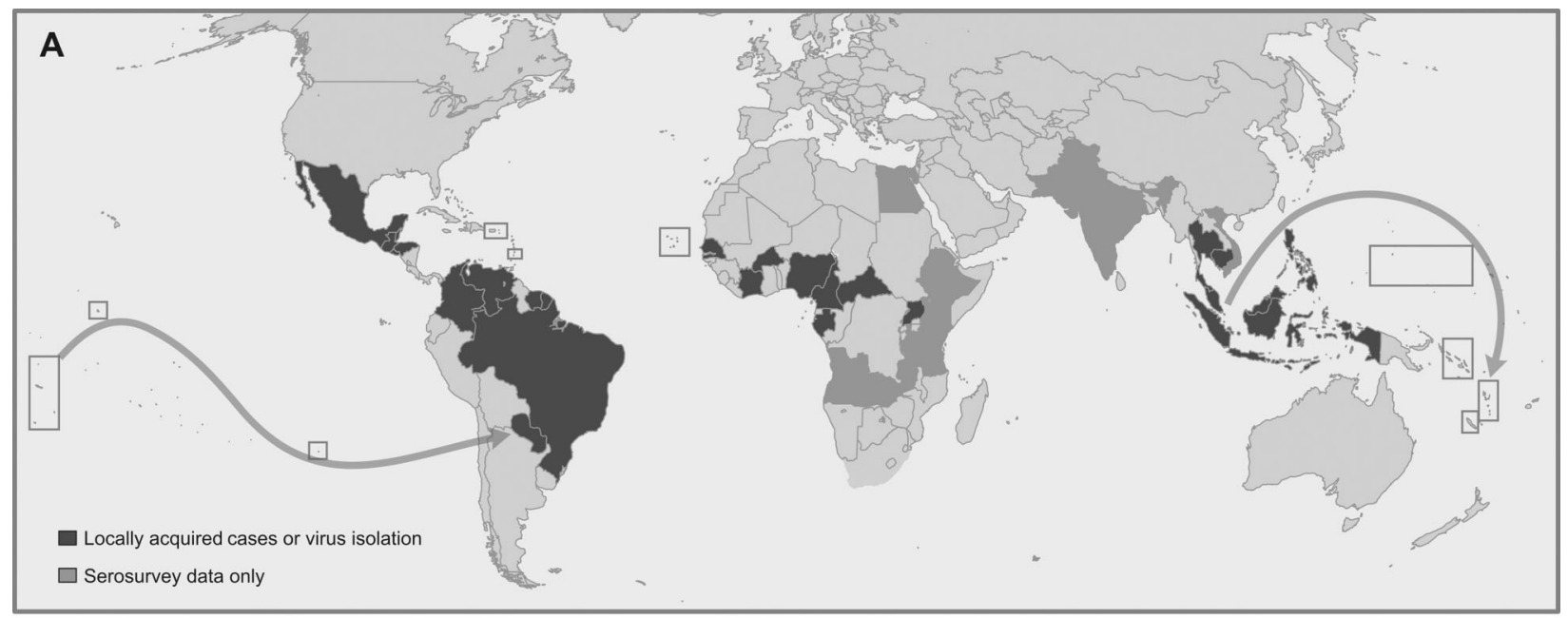

B

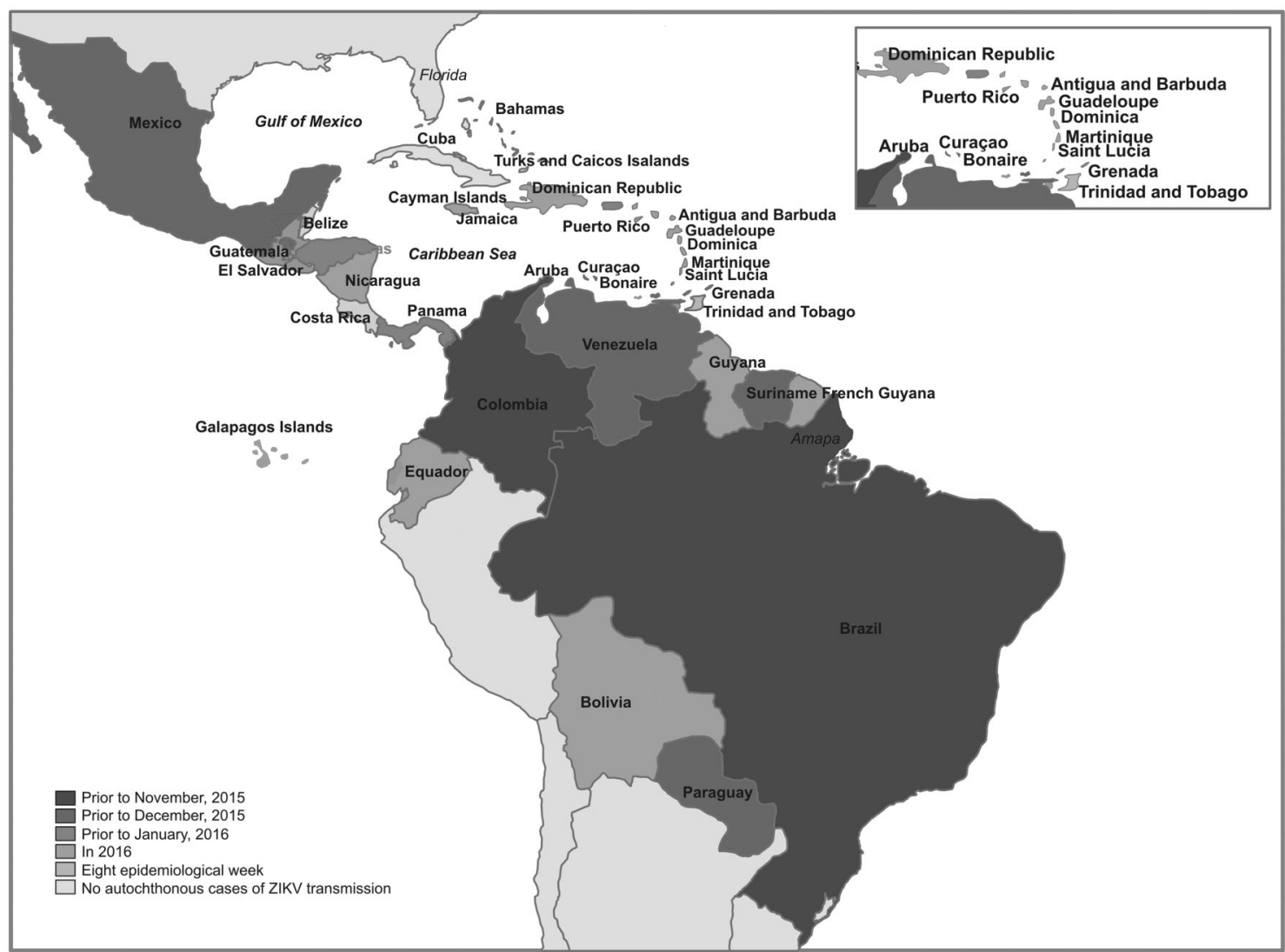

Figure 1. World epidemiology of ZIKV infection. A, Global expansion of the Asian lineage. After the characterization and isolation of ZIKV in Uganda, until 2007 only few human cases were reported in some African and Southeast Asiatic countries. The first significant ZIKV outbreak was registered in Yap Island, Micronesia, geographically situated close to Indonesia, where ZIKV is endemic (arrow). Between 2013 and 2014, ZIKV was rapidly spread to many of the islands of the Southern Pacific Ocean, causing the largest outbreak in French Polynesia. In 2015, the first autochthonous cases were detected in Brazil. B, Epidemiology of ZIKV infection in Central and South America. Currently, Brazil is the most affected country in South America followed by Colombia (maps adapted from the Pan-American World Health Organization, PAHO, Zika-epidemiological update). 


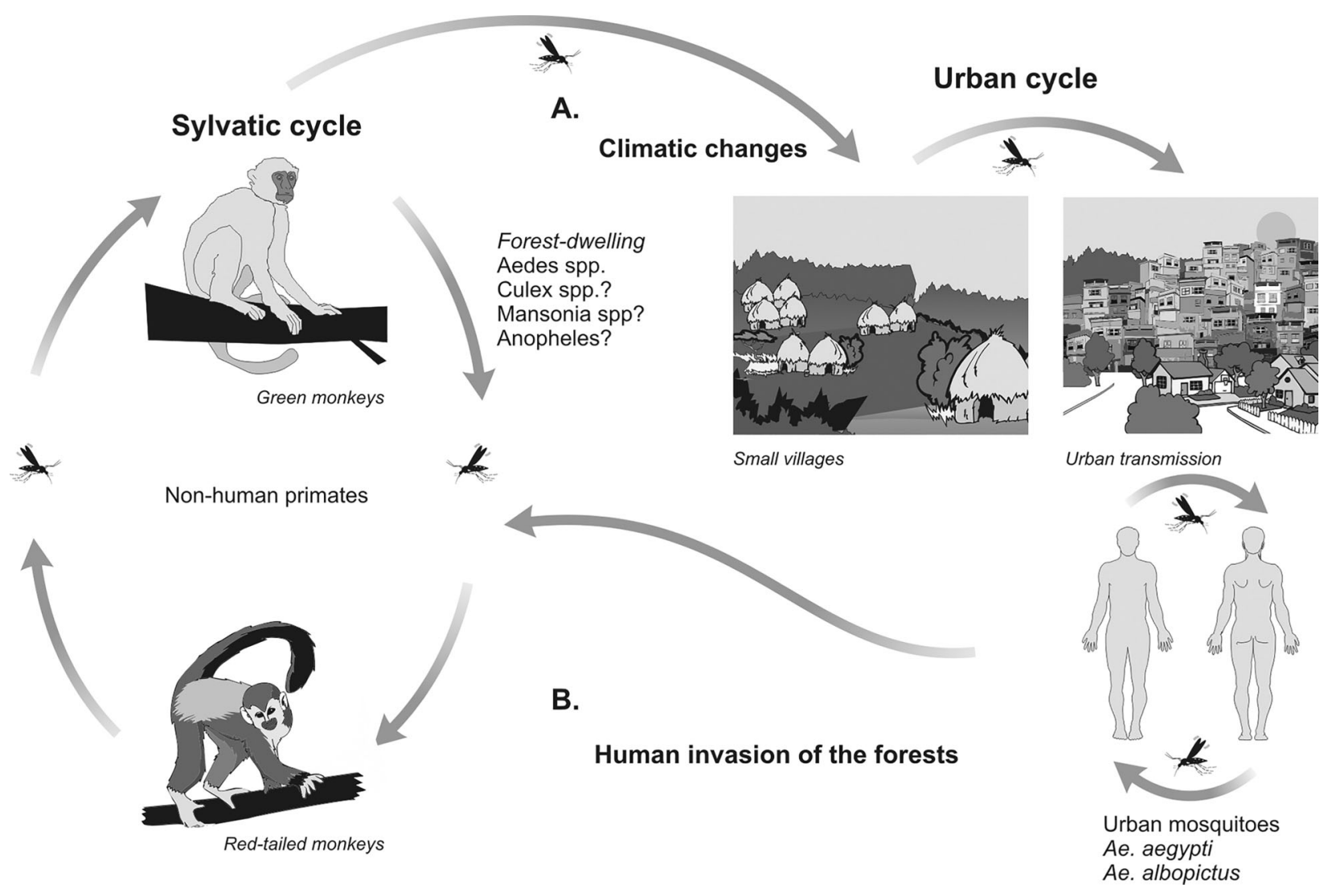

Figure 2. Transmission of ZIKV in its African sylvatic and urban cycles. In nature, it is thought that ZIKV infection is transmitted among non human primates with the help of different forest-dwelling mosquitoes, principally of the Aedes genus. It is unknown how the urban transmission occurs. Probably during heavy rainfalls, the sylvatic mosquito population can grow up progressively and disseminate the virus to nearby villages, and from there to larger urban centers, thus the urban cycle may occur with human-to-human transmission $(A)$. Another possible route for human-to-human ZIKV infection is the direct human invasion of the forest habitats, where the infection can be transmitted to human hosts from forest dwelling mosquitoes $(B)$.

These non-human primates live in a narrow strip in Central Africa. It is possible that they are natural ZIKV reservoirs, whilst the transmission of ZIKV between them is maintained by forest-dwelling mosquitoes.

The mosquito population can proliferate during heavy rainfalls, surpass the forest boundaries, and thus ZIKV can be spread to nearby villages. Consequently, the infection can be transmitted to local inhabitants, who can participate in the dissemination of ZIKV in larger urban centers. Another possibility for ZIKV transmission is the advance of human activities in the forests, and therefore, transmission of the infection directly to humans by forest-dwelling mosquitoes (Figure 2). A recent finding demonstrated that pools of $A e$. albopictus collected in the capital city of Gabon, Libreville, are positive for the ZIKV African lineage, strongly suggesting the participation of Ae. albopictus mosquitoes in the ZIKV urban cycle in Africa (25).

The epidemiologic situation of ZIKV in Asia shows some differences compared to Africa. ZIKV has been isolated from Ae. aegypti mosquitoes in Malaysia (4), however there is no information for another arthropod species, which can harbor ZIKV in this region. Because Ae. aegypti is a strict synanthropic mosquito, it is highly probable that it participates in the ZIKV urban cycles in Southeast Asia. This is additionally confirmed by the significant salivary susceptibility of urban Ae. aegypti captured in Singapore to the ZIKV African strain (26). The natural ZIKV reservoirs in Asia are unknown. It has been observed that orangutans (Pongo pygmaeus) from Borneo show high seroprevalence to different arbovirus infections, including ZIKV (27), and therefore, they may serve as ZIKV natural reservoirs in the Asiatic sylvatic cycle. Ae. hensilli, an abundant mosquito in the Islands of the Western Pacific Ocean, was suspected to be the predominant vector of the ZIKV Yap epidemic in 2007. However, no ZIKV RNA was detected in any of the mosquito pools collected on the island (28). Therefore, the participation of $A e$. hensilli as transmitting vector of ZIKV needs to be elucidated. Additionally, due to the unclear 
epidemiology of ZIKV infection, further laboratory and field studies are crucial in order to define the viral competence for other animal and arthropod hosts, including mosquito species from newly invaded areas like the South American countries.

Other modes of ZIKV transmission, including vertical, sexual and parenteral have also been proposed. The detection of ZIKV RNA in semen from a Tahiti man complaining from hematospermia during the ZIKV outbreak in French Polynesia (29), and the probable sexual transmission of ZIKV from a man to woman with sexual contact a few days before the onset of the man's symptoms (30) suggests that the ZIKV could be transmitted sexually. The probable relationship between ZIKV and fetal microcephaly proposes parenteral transmission and risk of severe congenital infection. There is evidence for such a transmission route. Initially, during the ZIKV epidemic in French Polynesia, evidence for perinatal transmission of ZIKV has been documented (31), as judged by the detection of ZIKV RNA in plasma of newborns. Another relationship suggesting ZIKV vertical transmission is the observed unusual increase of microcephaly cases in the Northeast part of Brazil. The Brazilian health authorities are suspicious of a connection between the microcephaly and ZIKV, as the increase is associated spatio-temporally to the ZIKV outbreak in the country (32). Detection of ZIKV sequences in fetuses with malformations (microcephaly, hidrancephaly, hydrops) strongly suggests ZIKV transplacental passage and capacity to cause congenital infection with neuronal damage (33-35). However, the temporal association between ZIKV and microcephaly is not sufficient to establish a "cause-effect" relationship. The cases with confirmed microcephaly and ZIKV RNA presence are very limited compared to the total number of cases with microcephaly. Even if it is supposed that ZIKV causes intrauterine infections, more studies are needed in order to define the pathogenic effect of ZIKV on the neuronal tissues, the outcome of the infection (post-natal abnormalities) and the epidemiological burden of ZIKV in the affected countries. Moreover, an increased prevalence of microcephaly could be due to multiple causes including other viruses and parasites, irradiation, toxic substances, intrauterine growth retardation and chromosomal disorders. All of these factors have to be discarded in order to establish a vertical transmission of ZIKV and potential for induction of birth defects or stillbirths.

\section{Diagnosis of ZIKV}

Up to this day, there is no officially approved commercial kit for ZIKV serological diagnosis. During the Yap ZIKV epidemic IgM-serological investigation on the collected samples was performed at the Arboviral Diagnostic and Reference Laboratory, Centers for Disease Control and Prevention (Atlanta, GA, USA) following a routine protocol for anti-arboviral IgM detection (36).
However, a significant proportion of the tested samples showed low levels of cross-reactivity for related flaviviruses (Japanese Encephalitis Virus, Yellow Fever Virus, West Nile Virus, Saint Louis Encephalitis Virus and Murray Valley Encephalitis Virus) and especially DENV (14). However, this result can be expected due to the complex serology of the Flaviviridae family resulting from the extensive crossreactivity between the anti-flavivirus antibodies and the wide distribution of immunity against these infections in the tropical regions. The existing ZIKV serological kits on the market (Zika Virus Rapid Test, Biocan, Canada; Human Zika Virus IgG ELISA Kit, MyBioSource, USA; Anti-Zika Virus ELISA lgG/lgM, Euroimmun, Germany) are only for research purposes and their cost does not justify application during epidemics in the tropical regions. Moreover, the diagnostic specificity of these tests for samples obtained from patients living in the Tropics can be doubtful as the population of such countries demonstrates high indices of Flavivirus immunity.

To avoid the unspecific results obtained by ELISA, molecular detection methods can be successfully applied for ZIKV diagnosis. The results of the molecular testing are rapid and in many cases can be quantitative. Many PCR modifications with differing sensitivities are available for ZIKV detection. The most widely used assays are based on real-time hydrolysis probes (14,37-39), however, conventional PCR methods are also available (23). ZIKV can be detected in different specimens: blood (plasma, serum), urine, saliva, and fetal tissues $(14,35,40,41)$. A disadvantage of the PCR performed on blood samples is that the duration of ZIKV viremia is relatively short and the virus can be detected only by the time of the appearance of clinical symptoms (14). On the contrary, the ZIKV shedding in urine can be more prolonged ( $\sim 15$ days after the disappearance of clinical symptoms) than the duration of viremia, and can present higher viral load. Therefore urine specimens can also be used for ZIKV confirmation $(40,42,43)$. Moreover, the collection of urine is a non-invasive procedure that makes this specimen interesting for ZIKV diagnosis, especially in children and newborns. During the ZIKV outbreak in French Polynesia the virus has been detected in saliva (41), which can also be used for ZIKV detection. However, it is unknown how long the liberation of viral particles in saliva lasts. Manipulation with this type of specimen can be tricky due to its insufficient volume and usually low viral quantities. We believe that for confirmation of ZIKV infection a combined approach must be applied including blood testing as a principal specimen for ZIKV RNA detection, and urine/saliva testing as additional markers for viral confirmation. On the contrary, for the confirmation of congenital ZIKV infection and the relationship of ZIKV with fetal abnormalities, the molecular test must be performed on fetal tissues (brain, placenta) $(35,44)$ or amniotic fluid (34).

For ZIKV confirmation, viral isolation on cell cultures is also possible. However, ZIKV isolation in cell cultures can be 
time consuming and is not suitable for routine diagnosis. $\mathrm{ZIKV}$ is best isolated after intracerebral inoculation of clinical materials into newborn mice (1). ZIKV can also be isolated on different cell lines including mosquito-derived (AP-61, Ae. pseudoscutellaris; C6/36, Ae. albopictus) or derived from non-human primates (VERO, kidney epithelial cells from African green monkey; BHK, baby hamster kidney cells) (22).

The diagnosis of ZIKV infection seems to be a complex issue. The principle sample for virus RNA detection can be plasma, however, it must be combined with the simultaneous ZIKV PCR on urine/saliva. For confirmation of congenital infection, PCR on amniotic fluid is plausible. Due to the short duration of the viremia, specimens must be collected immediately during the onset of the clinical disease. ZIKA serological confirmation is possible, despite its limited diagnostic significance and high cost.

\section{ZIKV clinical manifestations and treatment}

Although ZIKV infection is thought to be asymptomatic in approximately $80 \%$ of infected people, the virus causes a mild, non-specific and self-limiting infection resembling Chikungunya or DENV fevers (21). The duration of the incubation period is thought to last a few days. The classic clinical picture of ZIKV infection is manifested by low-grade fever $\left(37.8\right.$ to $\left.38.5^{\circ} \mathrm{C}\right)$, bilateral non-purulent conjunctivitis, headache, myalgia and arthralgia with periarticular edema of the small joints lasting for up to one week. ZIKV infection also causes a generalized, erythematous, maculopapular rash that spreads from the face to the limbs (21).

Patients with uncomplicated ZIKV disease are generally not treated, since the symptoms resolve within 3-7 days (21). The only available treatment for the infection is palliative. Fever and/or arthralgia can be treated with acetaminophen and the itching with anti-histamine drugs. It is also recommended an adequate rehydration due to fluid loss. Aspirin and nonsteroidal anti-inflammatory medicaments are not recommended due to the possibility of misdiagnosing the infection as DENV (21).

Despite the ZIKV classic mild illness, it is thought that this virus can also exert a pathological effect on neuronal tissues of a fetus or an adult. During the French Polynesia ZIKV outbreak (2013-2014), a 20-fold increase of GuillainBarré syndrome (GBS) was reported compared to previous years (32). Another evidence for ZIKV neurotropism is the reported case of a young girl with acute myelitis with a possible ZIKV etiology. Moreover, during the Brazilian ZIKV outbreak, the first associations of ZIKV and fetal neuronal malformations were reported. Although, no causal relationship between ZIKV infection and neurological damage has been established yet, there is significant evidence supporting such a hypothesis.

\section{Neurological non-congenital ZIKV effect}

GBS is characterized by progressive paralysis over 1-3 weeks due to an immune response, typically occurring after minor viral or bacterial infections. These include influenza, Campylobacter jejuni, cytomegalovirus and Epstein-Barr virus (45-47). Patients usually present affected motor function, beginning distally and progressing proximally. This includes bilateral weakness of the arms and legs, areflexia, sensory disturbances and involvement of the cranial nerves and muscles, in some cases affecting the eye movements or swallowing (48). These symptoms can last from few weeks to several months. The risk of GBS increases with age, and men are more commonly affected than women (49). The clinical outcome of GBS is related to a $5 \%$ death rate and $20 \%$ of the patients remain with significant disability (50).

The recent outbreaks of ZIKV demonstrated that this virus can be involved in the development of GBS. The first evidence of ZIKV-associated GBS was reported during the French Polynesia outbreak (51), in which 42 patients were diagnosed with subacute flaccid paralysis after ZIKV infection (52). Most of them (88\%) had shown classical ZIKV symptoms with onset of the neurological disease in about 6 days. The patients presented generalized muscle weakness, incapacity to walk, facial palsy and increased protein concentration in the cerebrospinal fluid (CSF). None of them demonstrated ZIKV RNA in the serum but presented anti-ZIKV IgM (93\%), IgG (69\%), and neutralizing antibodies (100\%) (52).

The treatment for ZIKV-induced GBS is only palliative. Approaches include application of intravenous immune globulins and in rare cases, plasmapheresis. The patients showed rapid recovery, and after 3 months $57 \%$ of them were able to walk without assistance. The GBS incidence during the French Polynesia ZIKV outbreak was estimated to be approximately $0.24 \%$ ZIKV infections (52).

Another neurological commitment recently related to ZIKV is the acute myelitis (inflammation of white/gray matter of the spinal cord). A patient from the Guadalupe Island presented a clinical picture of myelitis and severe pain. High ZIKV RNA load was detected in serum, urine and CSF nine days after symptoms onset. No other infectious agent was detected. The patient was treated with methylprednisolone and after 1 month the legs were still presenting moderate weakness (53). No relationship between myelitis and ZIKV infection has been established. However, the neurotropism of some flaviviruses such as DENV (54), Japanese encephalitis (55), and West Nile (56) causing encephalitis and myelitis is well documented, therefore, it is highly probable that ZIKV can also be involved as a causing agent in inflammatory neurological disease.

\section{Congenital ZIKV infection}

ZIKV infection has become an important worldwide public health issue since its association with microcephaly in neonates in Brazil (57).

Microcephaly is characterized by a smaller occipital frontal circumference of the head of any newborn, than expected for age, evident at birth (primary microcephaly) 
or postnatally (secondary microcephaly). There is no consensus in the scientific community for the diagnosis of fetal microcephaly, especially in the context of ZIKV infection (58). In general, during prenatal observations the measure of the occipital frontal circumference by ultrasound is compared to the fetal age-related mean, in order to establish how small it is in relation to the gestational age. There are different causes for microcephaly development, such as exposure to environmental factors (heavy metals, smoking, alcohol, radiation), genetic causes, maternal diabetes, and pathogens (cytomegalovirus, toxoplasmosis, rubella, herpes simplex, syphilis, HIV, and West Nile virus) (32). More recently, ZIKV was also associated with development of microcephaly (57).

According to the WHO, a newborn with head circumference equal to or lower than two standard deviations $(\leqslant 2 \mathrm{SD})$ below the mean is characterized as microcephalic, and below $3 S D$ is referred to as severe prognosis of microcephaly (59). The clinical findings in microcephaly include marked cerebral atrophy and ventriculomegaly, extensive intracranial calcifications, simplified gyral patterns, dysgenenis of the corpus collosum, and cerebellar hypoplasia (60). Consequently, it can be associated with intellectual disability, developmental delay and seizures. Microcephaly is a lifelong condition and there is no cure or standard treatment. However, it is worth noting that most fetuses diagnosed prenatally by ultrasound will not have pathological microcephaly at birth, despite their small head size.

A report from Paraiba, Brazil related two fetuses with microcephaly by prenatal ultrasound from two pregnant women who had ZIKV symptoms but were ZIKV RNA negative in blood, and ZIKV positive in amniotic fluid. One of the neonates was born with 40 weeks gestational age and head circumference almost 3SD below the mean. The other neonate was born with severe ventriculomegaly, microphtalmia, cataract and severe arthrogryposis of the legs and arms (61). Similar results were obtained by the study of Mlakar et al. (2016), where ZIKV positive fetus with microcephaly had almost complete agyria, internal hydrocephalus, and calcifications in the cortex and subcortical white matter in the frontal, parietal, and occipital lobes (35). Almost all of the case-reports demonstrate fetal abnormalities apart from microcephaly including multifocal intracranial and placental calcifications, ventriculomegaly, intrauterine growth restriction, brain atrophy, and other less frequent abnormalities $(21,35,44,58,62,63)$. Fetal abnormalities can be detected in up to $29 \%$ of ZIKV-positive pregnant women (62). Preliminary analyses demonstrate that the highest risk of microcephaly or congenital anomalies is during the first trimester of pregnancy $(57,58)$, however, ZIKV congenital abnormalities were observed in fetuses of women who were infected by ZIKV at any week of gestation (62). The severity of other neurological damages associated with congenital infections like rubella e cytomegalovirus is inversely related to the gestational period of the fetal infection (58). Thus, more data is needed to correlate fetal abnormalities to time of infection, once it could be related with severe sequelae in the newborn.

Fetal deaths and miscarriages have also been reported in ZIKV-positive pregnant women. The reported miscarriages occurred during the first trimester (11th and 13th weeks) $(44,62)$. Women who presented fetal deaths were infected with ZIKV during the second and third trimester, and presented at least maculopapular rash during the acute phase of infection (62). Newborn lethality within the first $20 \mathrm{~h}$ after birth from ZIKV infected mothers has also been reported (44).

Congenital ocular findings concomitant with microcephaly have also been associated with ZIKV infection during pregnancy. Ocular abnormalities were present in $34.5 \%$ of microcephalic infants examined, and involved the bilateral vision in $70 \%$ of them. The lesions included focal pigment mottling of the retina, chorioretinal athropy, optic nerve abnormalities, bilateral iris coloboma (congenital fissure) and lens dislocation. These lesions are considered vision threatening eye damage and also can be caused by West Nile virus infection, toxoplasmosis, cytomegalovirus, rubella, herpes simplex virus and syphilis. This study shows the importance of testing microcephalic newborns for ocular abnormalities, since the rate is reported to be very high during the ZIKV epidemic in Brazil (64).

Other tissues besides neuronal and optical may also be affected by ZIKV congenital infection. This association was demonstrated in a report from Salvador, Brazil, which showed a fetus miscarriage (32nd week) with microcephaly, hydranencephaly, intracranial calcifications and destructive lesions of posterior fossa. Hydrotorax, ascites and subcutaneous edema were also reported. It is suggested that the mother had an asymptomatic ZIKV infection during the 1 st trimester of gestation. ZIKV RNA was detected at cerebral cortex, medulla oblongata, cerebrospinal and amniotic fluid. Other organs did not present viral RNA (33).

\section{Lessons learned from the ZIKV epidemic in Brazil}

Currently, Brazil is experiencing the largest ZIKV epidemic among the countries of Latin America. Preliminary data announced on December 2015, by the European Centre for Disease Prevention and Control, estimate that between 440,000 and 1.3 million cases of autochthonous transmission of ZIKV have occurred in Brazil, but these data are largely underestimated and the real magnitude of the Brazilian ZIKV epidemic may be much higher (59).

The most significant problem in Brazil, concerning the ZIKV epidemic is the relationship between the virus and development of fetal abnormalities. The observations began in September 2015, when an increasing number of newborns with microcephaly was reported from the 
relatively small Pernambuco State, Northeast Brazil. This preliminary data demonstrated significant increase of this condition when compared to previous years and the governmental authorities started a national task force program for defining the cause. The first official report suggesting a relationship between ZIKV and congenital neurological malformations for the period between August-October 2015 was published early in 2016 (57). Consequently, ophthalmological abnormalities like macular atrophy and cerebral calcifications were attributed to ZIKV infection acquired during pregnancy, but without ZIKV RNA detection (65). The first possible relationship between ZIKV and development of microcephaly was reported for a pregnant Slovenian woman, who lived temporarily in Brazil and experienced febrile illness with rash at the end of the first trimester of pregnancy. The ultrasonography performed at 29 weeks of gestation revealed severely affected fetal central nervous system with calcifications and gross intrauterine growth retardation. In the aborted fetus, ZIKV RNA was detected uniquely in fetal brain tissues with high viral load $\left(6.5 \times 10^{7}\right.$ copies/mg tissue) and the complete genome of the infected strain was sequenced. The phylogenetic analysis showed close resemblance to the Asian clade. More recently, studies performed in Brazil found a more direct relationship between ZIKV infection and microcephaly as confirmed by the detection of ZIKV genome in the amniotic fluid of pregnant women (34) and in newborns with severe birth defects (33). A prospective study performed between September, 2015 and February, 2016 in pregnant women who tested positive for ZIKV in blood/ urine demonstrated fetal abnormalities in 12 of 42 fetuses (62). Moreover, a study examining ZIKV effect on human neural progenitor cells demonstrated that the infection increases cell death and dysregulates cell-cycle progression resulting in attenuated cell growth (66). These results suggest that ZIKV can be involved in the development of congenital infection, which can be responsible for the appearance of fetal abnormalities, especially in the central nervous system. Up to February 27, 2016 according to the Brazilian Ministry of Health, 5,640 cases of microcephaly in the country have been reported, and 583 of them are confirmed to have microcephaly and/or other central nervous system findings, suggestive of congenital infection. Of the total cases with confirmed microcephaly, only 67 fulfill the laboratory criteria for ZIKV infection, and 4,107 across Brazil remain under investigation (67). Although, there has been evidence that ZIKV can cause severe congenital infection, many questions remain unanswered. The most important are, how the virus reaches the fetus, what is the ZIKV impact during the different periods of formation of the fetus, and if the virus is detected long after acute infection in fetal brain tissues, what are the sites of viral persistence in the fetus.
The burden of microcephaly cases in Brazil poses serious problems for the health authorities, not only due to the high cost of the medical care involved in the assistance of the families, but also to the fact that the majority of the cases is clustered in the poorest regions of Brazil with precarious healthcare. The difficulty arises also from the difficulty in controlling the population of the presumable transmitting vector Ae. aegypti in the extensive national territory of Brazil, despite the involvement of the Brazilian Army. Despite the measures taken, the epidemic potential of ZIKV shows that Brazil is not prepared to deal with large urban outbreaks of tropical viral diseases, and organized efforts are necessary not only to control the vectors population, but also to educate the people about the clinical impact of the arboviral diseases. More investments are also necessary to demonstrate the scientific link between ZIKV infection and development of fetal neuronal abnormalities, and to develop a vaccine for the immunization of the general population.

\section{Conclusion}

The emergence of ZIKV in Brazil with the involvement of a large number of cases shows that arboviral infections can easily cross international frontiers and have serious impact in countries that have no preparedness or programs to deal with extensive outbreaks. Moreover, the probable relationship between ZIKV and congenital microcephaly in Brazil poses a great burden at the national health system of the country, which would have to be involved in the support of the affected children for their lifetime. Despite the fragmented reports of various Brazilian scientific research groups regarding the pathogenesis of ZIKV infection, there is no scientific unification to study in more details the transmission, the clinical impact and the possibility of treatment of this flaviviral infection. Strategies for the development of a vaccine are also imperative. Similarly, a serological test to perform a robust serologic survey throughout the national territory to evaluate the impact and epidemiology of ZIKV is urgently needed. Better understanding of the natural history of ZIKV infection is needed in order to establish effective control measures for this outbreak in Brazil and Latin America.

\section{Acknowledgments}

We are grateful to Sandra Navarro Bresciani for the artwork. We are also grateful to the funding agencies Fundação de Amparo e Pesquisa do Estado de São Paulo-FAPESP, Brazil (\#2009/16623-1, CTC-\#1998/14. 247-6 and INCTC-\#2008/57.877-3), and the Conselho Nacional do Desenvolvimento Científico e Tecnológico, Brazil (INCTC-\#573.754/2008-0). 


\section{References}

1. Dick GW, Kitchen SF, Haddow AJ. Zika virus. I. Isolations and serological specificity. Trans $R$ Soc Trop Med Hyg 1952; 46: 509-520. Available at: http://www.ncbi.nlm.nih.gov/ pubmed/12995440, doi: 10.1016/0035-9203(52)90042-4

2. Macnamara FN. Zika virus: a report on three cases of human infection during an epidemic of jaundice in Nigeria. Trans $R$ Soc Trop Med Hyg 1954; 48: 139-145. Available at: http:// www.ncbi.nlm.nih.gov/pubmed/13157159, doi: 10.1016/00359203(54)90006-1.

3. Haddow AJ, Williams MC, Woodall JP, Simpson DI, Goma LK. Twelve isolations of Zika virus from Aedes (Stegomyia) Africanus (Theobald) taken in and above a Uganda forest. Bull World Health Organ 1964; 31: 57-69.

4. Marchette NJ, Garcia R, Rudnick A. Isolation of Zika virus from Aedes aegypti mosquitoes in Malaysia. Am J Trop Med Hyg 1969; 18: 411-415. Available at: http://www.ncbi.nlm. nih.gov/pubmed/4976739.

5. Geser A, Henderson BE, Christensen S. A multipurpose serological survey in Kenya. 2. Results of arbovirus serological tests. Bull World Health Organ 1970; 43: 539-552. Available at: http://www.ncbi.nlm.nih.gov/pubmed/5313066.

6. Robin Y, Mouchet J. [Serological and entomological study on yellow fever in Sierra Leone]. Bull Soc Pathol Exot Filiales 1975; 68: 249-258.

7. Jan C, Languillat G, Renaudet J, Robin Y. [A serological survey of arboviruses in Gabon]. Bull Soc Pathol Exot Filiales 1978; 71: 140-146. Available at: http://www.embase.com/search/ results?subaction=viewrecord\&from $=$ export\&id=L9070629.

8. Saluzzo JF, Ivanoff B, Languillat G, Georges AJ. [Serological survey for arbovirus antibodies in the human and simian populations of the South-East of Gabon (author's transl)]. Bull Soc Pathol Exot Filiales 1982; 75: 262-266. Available at: http://www.ncbi.nlm.nih.gov/pubmed/6809352.

9. Chippaux A, Chippaux-Hyppolite C, Monteny-Vandervorst $\mathrm{N}$, Souloumiac-Deprez D. [Several yellow fever cases in an endemic area in Ivory Coast: serological and epidemiological evidence (author's transl)]. Med Trop 1981; 41: 53-61.

10. Saluzzo JF, Gonzalez JP, Herve JP, Georges AJ. [Serological survey for the prevalence of certain arboviruses in the human population of the south-east area of Central African Republic (author's transl)]. Bull Soc Pathol Exot Filiales 1981; 74: 490499. Available at: http://www.ncbi.nlm.nih.gov/pubmed/6274526.

11. Monlun E, Zeller H, Le Guenno B., Traore-Lamizana M, Hervy JP, Adam F, et al. [Surveillance of the circulation of arbovirus of medical interest in the region of eastern Senegal]. Bull Soc Pathol Exot 1993; 86: 21-28. Available at: http://www.ncbi.nlm.nih.gov/pubmed/8099299.

12. Olson JG, Ksiazek TG, Suhandiman, Triwibowo. Zika virus, a cause of fever in Central Java, Indonesia. Trans $R$ Soc Trop Med Hyg 1981; 75: 389-393, doi: 10.1016/0035-9203 (81)90100-0.

13. Olson JG, Ksiazek TG, Gubler DJ, Lubis SI, Simanjuntak G, Lee $\mathrm{VH}$, et al. A survey for arboviral antibodies in sera of humans and animals in Lombok, Republic of Indonesia. Ann Trop Med Parasitol 1983; 77: 131-137. Available at: http:// www.ncbi.nlm.nih.gov/pubmed/6309104.

14. Lanciotti RS, Kosoy OL, Laven JJ, Velez JO, Lambert AJ, Johnson AJ, et al. Genetic and serologic properties of Zika virus associated with an epidemic, Yap State, Micronesia,
2007. Emerg Infect Dis 2008; 14: 1232-1239. Available at: http://www.ncbi.nlm.nih.gov/pubmed/18680646, doi: 10.3201/ eid1408.080287

15. Cao-Lormeau VM, Roche C, Teissier A, Robin E, Berry AL, Mallet HP, et al. Zika virus, French polynesia, South pacific, 2013. Emerg Infect Dis 2014; 20: 1085-1086. Available at: http://www.ncbi.nlm.nih.gov/pubmed/24856001.

16. Musso D, Nilles EJ, Cao-Lormeau VM. Rapid spread of emerging Zika virus in the Pacific area. Clin Microbiol Infect 2014; 20: O595-O596, doi: 10.1111/1469-0691.12707.

17. Zanluca C, Melo VC, Mosimann AL, Santos GI, Santos CN, Luz K. First report of autochthonous transmission of Zika virus in Brazil. Mem Inst Oswaldo Cruz 2015; 110: 569-572. Available at: http://www.ncbi.nlm.nih.gov/pubmed/26061233, doi: 10.1590/0074-02760150192.

18. Musso D. Zika Virus Transmission from French Polynesia to Brazil. Emerg Infect Dis 2015; 21: 1887, doi: 10.3201/ eid2110.151125.

19. Cook S, Holmes EC. A multigene analysis of the phylogenetic relationships among the flaviviruses (Family: Flaviviridae) and the evolution of vector transmission. Arch Virol 2006; 151: 309-325. Available at: http://www.ncbi.nlm.nih. gov/pubmed/16172840, doi: 10.1007/s00705-005-0626-6.

20. Faye $O$, Freire CC, lamarino A, Faye O, de Oliveira JV, Diallo $\mathrm{M}$, et al. Molecular evolution of Zika virus during its emergence in the 20(th) century. PLoS Negl Trop Dis 2014; 8: e2636, doi: 10.1371/journal.pntd.0002636.

21. Chan JF, Choi GK, Yip CC, Cheng VC, Yuen KY. Zika fever and congenital Zika syndrome: An unexpected emerging arboviral disease. J Infect 2016. Available at: http:// linkinghub.elsevier.com/retrieve/pii/S016344531600061X.

22. Haddow AD, Schuh AJ, Yasuda CY, Kasper MR, Heang V, Huy R, et al. Genetic characterization of Zika virus strains: geographic expansion of the Asian lineage. PLoS Negl Trop Dis 2012; 6: e1477, doi: 10.1371/journal.pntd.0001477.

23. Faye O, Faye O, Dupressoir A, Weidmann M, Ndiaye M, Alpha SA. One-step RT-PCR for detection of Zika virus. J Clin Virol 2008; 43: 96-101, doi: 10.1016/j.jcv.2008.05.005.

24. Marcondes CB, Ximenes MF. Zika virus in Brazil and the danger of infestation by Aedes (Stegomyia) mosquitoes. Rev Soc Bras Med Trop 2016; 49: 4-10, doi: 10.1590/00378682-0220-2015.

25. Grard G, Caron M, Mombo IM, Nkoghe D, Mboui OS, Jiolle D, et al. Zika virus in Gabon (Central Africa) - 2007: a new threat from Aedes albopictus? PLoS Negl Trop Dis 2014; 8: e2681. Available at: http://www.ncbi.nlm.nih.gov/pubmed/ 24516683, doi: 10.1371/journal.pntd.0002681.

26. Wong PS, Li MZ, Chong CS, Ng LC, Tan CH. Aedes (Stegomyia) albopictus (Skuse): a potential vector of Zika virus in Singapore. PLoS Negl Trop Dis 2013; 7: e2348. Available at: http://www.ncbi.nlm.nih.gov/pubmed/23936579.

27. Wolfe ND, Kilbourn AM, Karesh WB, Rahman HA, Bosi EJ, Cropp BC, et al. Sylvatic transmission of arboviruses among Bornean orangutans. Am J Trop Med Hyg 2001; 64: 310-316.

28. Duffy MR, Chen TH, Hancock WT, Powers AM, Kool JL, Lanciotti RS, et al. Zika virus outbreak on Yap Island, Federated States of Micronesia. N Engl J Med 2009; 360: 2536-2543, doi: 10.1056/NEJMoa0805715. 
29. Musso D, Roche C, Robin E, Nhan T, Teissier A, Cao-Lormeau VM. Potential sexual transmission of Zika virus. Emerg Infect Dis 2015; 21: 359-361, doi: 10.3201/ eid2102.141363.

30. Foy BD, Kobylinski KC, Chilson Foy JL, Blitvich BJ, Travassos da RA, Haddow AD, et al. Probable non-vectorborne transmission of Zika virus, Colorado, USA. Emerg Infect Dis 2011; 17: 880-882, doi: 10.3201/eid1705.101939.

31. Besnard M, Lastere S, Teissier A, Cao-Lormeau V, Musso D. Evidence of perinatal transmission of Zika virus, French Polynesia, December 2013 and February 2014. Euro Surveill 2014; 19

32. Garcia E, Yactayo S, Nishino K, Millot V, Perea W, Briand S. Zika virus infection: global update on epidemiology and potentially associated clinical manifestations. Wkly Epidemiol Rec/Heal Sect Secr Leag Nations 2016; 91: 73-81. Available at: http://www.ncbi.nlm.nih.gov/pubmed/26897760.

33. Sarno M, Sacramento GA, Khouri R, do Rosario MS, Costa $F$, Archanjo $G$, et al. Zika virus infection and stillbirths: A case of hydrops fetalis, hydranencephaly and fetal demise. PLoS Negl Trop Dis 2016; 10: e0004517. Available at: http:// www.ncbi.nlm.nih.gov/pubmed/26914330, doi: 10.1371/journal. pntd. 0004517.

34. Calvet G, Aguiar RS, Melo AS, Sampaio SA, de Filippis I, Fabri $A$, et al. Detection and sequencing of Zika virus from amniotic fluid of fetuses with microcephaly in Brazil: a case study. Lancet Infect Dis 2016. Available at: http://linkinghub. elsevier.com/retrieve/pii/S1473309916000955.

35. Mlakar J, Korva M, Tul N, Popovic M, Poljsak-Prijatelj M, Mraz J, et al. Zika Virus Associated with Microcephaly. N Engl J Med 2016; 374: 951-958, doi: 10.1056/NEJMoa1600651.

36. Martin DA, Muth DA, Brown T, Johnson AJ, Karabatsos N, Roehrig JT. Standardization of immunoglobulin M capture enzyme-linked immunosorbent assays for routine diagnosis of arboviral infections. J Clin Microbiol 2000; 38: 1823-1826.

37. Faye O, Faye O, Diallo D, Diallo M, Weidmann M, Sall AA. Quantitative real-time PCR detection of Zika virus and evaluation with field-caught mosquitoes. Virol J 2013; 10: 311. Available at: http://www.virologyj.com/content/10/1/311, doi: 10.1186/1743-422X-10-311.

38. Tappe D, Rissland J, Gabriel M, Emmerich P, Gunther S, Held G, et al. First case of laboratory-confirmed Zika virus infection imported into Europe, November 2013. Euro Surveill 2014; 19: Available at: http://www.ncbi.nlm.nih.gov/ pubmed/24507467.

39. Pyke AT, Daly MT, Cameron JN, Moore PR, Taylor CT, Hewitson GR, et al. Imported zika virus infection from the cook Islands into Australia, 2014. PLoS Curr 2014; 6: Available at: http://www.pubmedcentral.nih.gov/articlerender. fcgi?artid=4055592\&tool=pmcentrez\&rendertype=abstract.

40. Gourinat AC, O’Connor O, Calvez E, Goarant C, DupontRouzeyrol M. Detection of Zika virus in urine. Emerg Infect Dis 2015; 21: 84-86, doi: 10.3201/eid2101.140894.

41. Musso D, Roche C, Nhan TX, Robin E, Teissier A, Cao-Lormeau VM. Detection of Zika virus in saliva. J Clin Virol 2015; 68: 53-55. Available at: http://www.ncbi.nlm.nih. gov/pubmed/26071336, doi: 10.1016/j.jcv.2015.04.021.

42. de M Campos R, Cirne-Santos C, Meira GL, Santos LL, de Meneses MD, Friedrich J, et al. Prolonged detection of Zika virus RNA in urine samples during the ongoing Zika virus epidemic in Brazil. J Clin Virol 2016; 77: 69-70. Available at: http://www.ncbi.nlm.nih.gov/pubmed/26921737, doi: 10.1016/ j.jcv.2016.02.009.

43. Shinohara K, Kutsuna S, Takasaki T, Moi ML, Ikeda M, Kotaki A, et al. Zika fever imported from Thailand to Japan, and diagnosed by PCR in the urines. J Travel Med 2016; 23: Available at: http://www.ncbi.nlm.nih.gov/pubmed/26782128.

44. Martines RB, Bhatnagar J, Keating MK, Silva-Flannery L, Muehlenbachs A, Gary J, et al. Notes from the field: evidence of zika virus infection in brain and placental tissues from two congenitally infected newborns and two fetal losses - Brazil, 2015. MMWR Morb Mortal Wkly Rep 2016; 65: 159-160. Available at: http://www.ncbi.nlm.nih. gov/pubmed/26890059, doi: 10.15585/mmwr.mm6506e1.

45. Tam CC, O'Brien SJ, Petersen I, Islam A, Hayward A, Rodrigues LC. Guillain-Barre syndrome and preceding infection with campylobacter, influenza and Epstein-Barr virus in the general practice research database. PLoS One 2007; 2: e344. Available at: http://www.ncbi.nlm.nih.gov/ pubmed/17406668, doi: 10.1371/journal.pone.0000344.

46. Lehmann HC, Hartung HP, Kieseier BC, Hughes RA. GuillainBarre syndrome after exposure to influenza virus. Lancet Infect Dis 2010; 10: 643-651. Available at: http://www.ncbi.nlm.nih. gov/pubmed/20797646, doi: 10.1016/S1473-3099(10)70140-7.

47. Grimaldi-Bensouda L, Alperovitch A, Besson G, Vial C, Cuisset JM, Papeix C, et al. Guillain-Barre syndrome, influenzalike illnesses, and influenza vaccination during seasons with and without circulating $\mathrm{A} / \mathrm{H} 1 \mathrm{~N} 1$ viruses. $A m$ J Epidemiol 2011; 174: 326-335. Available at: http://www. ncbi.nlm.nih.gov/pubmed/21652600, doi: 10.1093/aje/kwr072.

48. Ang CW, Jacobs BC, Laman JD. The Guillain-Barre syndrome: a true case of molecular mimicry. Trends Immunol 2004; 25: 61-66. Available at: http://www.ncbi.nlm.nih.gov/ pubmed/1510236, doi: 10.1016/j.it.2003.12.004.

49. McGrogan A, Madle GC, Seaman HE, de Vries CS. The epidemiology of Guillain-Barre syndrome worldwide. A systematic literature review. Neuroepidemiology 2009; 32: 150-163. Available at: http://www.ncbi.nlm.nih.gov/ pubmed/19088488, doi: 10.1159/000184748.

50. Yuki N, Hartung HP. Guillain-Barre syndrome. N Engl J Med 2012; 366: 2294-2304. Available at: http://www.ncbi.nlm.nih. gov/pubmed/22694000, doi: 10.1056/NEJMra1114525.

51. Oehler E, Watrin L, Larre P, Leparc-Goffart I, Lastere S, Valour $F$, et al. Zika virus infection complicated by GuillainBarre syndrome - case report, French Polynesia, December 2013. Euro Surveill 2014; 19: 7-9. Available at: http://www. ncbi.nlm.nih.gov/pubmed/24626205, doi: 10.2807/1560-7917. ES2014.19.9.20720.

52. Cao-Lormeau VM, Blake A, Mons S, Lastere S, Roche C, Vanhomwegen J, et al. Guillain-Barre syndrome outbreak associated with Zika virus infection in French Polynesia: a case-control study. 2016, doi: 10.1016/S0140-6736(16)00562-6.

53. Mecharles S, Herrmann C, Poullain P, Tran TH, Deschamps $\mathrm{N}$, Mathon $\mathrm{G}$, et al. Acute myelitis due to Zika virus infection. Lancet 2016. Available at: http://www.ncbi.nlm.nih.gov/ pubmed/26946926.

54. Larik A, Chiong Y, Lee LC, Ng YS. Longitudinally extensive transverse myelitis associated with dengue fever. BMJ Case Rep 2012; 2012: Available at: http://www.ncbi.nlm.nih.gov/ pubmed/22605876.

55. Verma R, Praharaj HN, Patil TB, Giri P. Acute transverse myelitis following Japanese encephalitis viral infection: an 
uncommon complication of a common disease. BMJ Case Rep 2012; 2012: Available at: http://www.ncbi.nlm.nih.gov/ pubmed/23008381.

56. Ali M, Safriel Y, Sohi J, Llave A, Weathers S. West Nile virus infection: MR imaging findings in the nervous system. AJNR Am J Neuroradiol 2005; 26: 289-297. Available at: http:// www.ncbi.nlm.nih.gov/pubmed/15709126.

57. Schuler-Faccini L, Ribeiro EM, Feitosa IM, Horovitz DD, Cavalcanti DP, Pessoa A, et al. Possible Possible association between Zika virus infection and microcephaly - Brazil, 2015. MMWR Morb Mortal Wkly Rep 2016; 65: 59-62. Available at: http://www.ncbi.nlm.nih.gov/pubmed/26820244, doi: $10.15585 / \mathrm{mmwr} \cdot \mathrm{mm} 6503 \mathrm{e} 2$.

58. Marrs C, Olson G, Saade G, Hankins G, Wen T, Patel J, et al. Zika virus and pregnancy: a review of the literature and clinical considerations. Am J Perinatol 2016. Available at: http://www.ncbi.nlm.nih.gov/pubmed/26939047.

59. Coulombier D, Danielsson N, Donachie A, Jansà J, Sudre B, Bortel W Van, et al. Rapid risk assesment ECDC internal response team Internal experts in alphabetical order: External experts consulted and acknowledgements. 2015.

60. Costa F, Sarno M, Khouri R, Freitas BDP, Siqueira I. Ideas and opinions emergence of congenital Zika syndrome: viewpoint from the front. 2016.

61. Martines RB, Bhatnagar J, Keating MK, Silva-Flannery L, Muehlenbachs A, Gary J, et al. Notes from the Field: Evidence of Zika virus infection in brain and placental tissues from two congenitally infected newborns and two fetal losses - Brazil, 2015. MMWR Morb Mortal Wkly Rep 2016; 65: 159-160, doi: 10.15585/mmwr.mm6506e1.
62. Brasil P, Pereira JP Jr, Raja GC, Damasceno L, Wakimoto M, Ribeiro Nogueira RM, et al. Zika virus infection in pregnant women in Rio de Janeiro - Preliminary Report. N Engl J Med 2016. Available at: http://www.ncbi.nlm.nih. gov/pubmed/26943629.

63. Oliveira Melo AS, Malinger G, Ximenes R, Szejnfeld PO, Alves SS, Bispo de Filippis AM. Zika virus intrauterine infection causes fetal brain abnormality and microcephaly: tip of the iceberg? Ultrasound Obstet Gynecol 2016; 47: 6-7. Available at: http://www.ncbi.nlm.nih.gov/pubmed/ 26731034, doi: 10.1002/uog.15831.

64. de Paula Freitas B, de Oliveira Dias J, Prazeres J, Sacramento GA, Ko Al, Maia M, et al. Ocular findings in infants with microcephaly associated with presumed zika virus congenital infection in Salvador, Brazil. JAMA Ophthalmol 2016; 1-19.

65. Ventura CV, Maia M, Bravo-Filho V, Gois AL, Belfort R, Jr. Zika virus in Brazil and macular atrophy in a child with microcephaly. Lancet 2016; 387: 228. Available at: http:// www.ncbi.nlm.nih.gov/pubmed/26775125, doi: 10.1016/S01406736(16)00006-4.

66. Tang H, Hammack C, Ogden SC, Wen Z, Qian X, Li Y, et al. Zika virus infects human cortical neural progenitors and attenuates their growth. Cell Stem Cell 2016; 1-4, doi: 10.1016/j.stem.2016.02.016.

67. European Centre for Disease Prevention and Control. Communicable disease threats report, 21-27 February 2016, week 8. Commun Dis Threat Rep 2016. Available at: http://ecdc.europa.eu/en/publications/Publications/communicable-disease-threats-report-27-feb-2016.pdf. 